\section{Oxidative stress in diabetic neuropathy: source of reactive oxygen species}

\author{
Mohit Chopra, Kirti Kaul, Joanna Tarr, \\ Ruhul Choudhury, Eva M. Kohner, \\ Rakesh Chibber \\ Institute of Biomedical and Clinical \\ Science, Peninsula College of Medicine \\ and Dentistry, University of Exeter, \\ St Luke's Campus, Exeter, UK
}

\section{Abstract}

Diabetic neuropathy is a common diabetic complication. Oxidative stress (OS) is a significant mediator in the development of diabetic neuropathy. Exposing neurons to hyperglycaemia increases OS, inhibits neurite outgrowth and causes apoptosis. In the present study we explore the role of nicotinamide adenine dinucleotide phosphate (NADPH) oxidase vs. mitochondrial reactive oxygen species (mtROS) in diabetic neuropathy. Human neuroblastoma SH-SY5Y cells were treated with high glucose $(25 \mathrm{mM})$ or normal glucose $(5.5$ $\mathrm{mM})$ in the presence and absence of the specific NADPH oxidase inhibitor gp91ds-tat (6 days). Caspase activity, intracellular ROS, mtROS production, mitochondrial membrane potential $(\Delta \Psi \mathrm{m})$ and NADPH oxidase activity were measured. Neurite outgrowth and neurites/cell were analysed using Image $\mathbf{J}$ software. Chronic exposure to high glucose increased caspases activity and cytoplasmic but not mtROS levels, and reduced the number of neurites/cell and the neurite outgrowth compared to normal glucose. Co-treatment with gp91ds-tat reversed the increased levels of ROS and prevented glucose-induced neurite inhibition. $\Delta \Psi \mathrm{m}$ and mtROS levels were unaffected at the end of the treatment. The current study demonstrates that NADPH oxidase, and not the mitochondria derived ROS, is responsible for the induction of apoptosis in SH-SY5Y cells. The use of NADPH oxidase inhibitors may prove to be of therapeutic value in the treatment of diabetic neuropathy.

\section{Introduction}

Diabetes is a world epidemic with figures suggesting that more than 366 million people are currently living with diabetes and this figure is expected to rise to 552 million by 2030 . At least $50 \%$ of these patients will develop neuropathy, a leading cause of non-traumatic lower limb amputations and autonomic failure in dia- betic patients. ${ }^{1}$ The natural progression of diabetic neuropathy involves nerve fiber loss, demyelination and axonal degeneration of myelinated fibres. ${ }^{2}$ The vast majority of patients with clinical diabetic neuropathy have a distal symmetrical form of the disorder that progresses following a fiber-length-dependent pattern, with sensory and autonomic manifestations predominating. Patients with long-standing diabetes mellitus, develop focal and multifocal neuropathies that include cranial nerve involvement and limb and truncal neuropathies. ${ }^{3}$

Although, the pathogenesis of diabetic neuropathy remains unclear, the Diabetic Control and Complications Trial and the United Kingdom Prospective Diabetes Study have confirmed the central role of hyperglycaemia in the disease process. 4,5 The major pathways of glucose metabolism that have been implicated in the development of microvascular complications include, i) increased flux through the polyol pathway, ii) non-enzymatic glycation of proteins yielding to the formation of advanced glycation end-products (AGE's), iii) activation of Protein Kinase C (PKC) and iv) increased hexosamine pathway activity. An increased production of reactive oxygen species (ROS) such as superoxide radical $\left(\mathrm{O}_{2}^{-}\right)$and hydroxyl radical $(\mathrm{OH} \cdot)$, has been suggested as a unified process that links the above pathways of hyperglycaemia induced damage. 6 There is evidence from various studies suggesting that increased production of ROS and oxidative stress leads to both the development and progression of diabetic neuropathy.7,8 Exposing neurons to elevated glucose increases oxidative stress which induces the production of ROS that triggers a mechanism that leads to apoptosis and inhibits neurite outgrowth.,910 The lack of normal neurite outgrowth might reflect the dysfunction of molecules or proteins that are important for maintaining a normal neuronal process. ${ }^{11}$

Oxidative stress is the imbalance between oxidants and antioxidants in favour of the oxidants, potentially leading to damage. Numerous enzyme systems produce ROS in mammalian cells, including; nicotinamide adenine dinucleotide phosphate (NADPH) oxidase, ${ }^{12}$ uncoupled nitric oxide (NO) synthase and the mitochondrial electron transport chain. ${ }^{13,14} \mathrm{~A}$ mitochondrial-dependent process has been suggested as a central mediator of oxidative stress in diabetic complications. ${ }^{15}$ We have recently exhibited that NADPH oxidase and not mitochondria - derived ROS is responsible for the accelerated apoptosis of pericytes in diabetic retinopathy (DR).16 Retinal capillary pericytes pre-treated with a NADPH oxidase inhibitor, apocynin reversed glucose-induced apoptosis, ROS production and $\mathrm{N}_{\varepsilon}$-(carboxymethyl) lysine (CML) production. ${ }^{16}$ The role of NADPH oxidase has been previously demonstrated in DR and diabetic nephropathy.17,18 NADPH oxidase has been shown to be responsible for the ROS pro-
Correspondence: Rakesh Chibber, Institute of Biomedical and Clinical Science, Peninsula College of Medicine and Dentistry, University of Exeter, St Luke's Campus, Magdalen Road, Exeter, EX1 2LU, UK. Tel. +44.01392.726336.

E-mail: rakesh.chibber@pms.ac.uk

Key words: diabetic neuropathy, oxidative stress, NADPH oxidase.

Acknowledgements: this study was supported in part by the PCMD Foundation and Diabetes and Related Diseases Research (DRDR) Association.

Contributions: MC designed the experiments, conducted the experiments, wrote and reviewed the manuscript; KK designed the experiments, conducted the experiments, reviewed the manuscript; JT reviewed the manuscript; RuC contributed to experiments, reviewed the manuscript; EK reviewed the manuscript; $\mathrm{RaC}$ proposed the hypothesis, designed the experiments, reviewed the manuscript, grant holder.

Conflict of interests: the authors report no conflict of interests.

Received for publication: 13 September 2012. Accepted for publication: 14 September 2012.

This work is licensed under a Creative Commons Attribution NonCommercial 3.0 License (CC BYNC 3.0)

(C) Copyright M. Chopra et al., 2012

Licensee PAGEPress, Italy

Endocrinology Studies 2012; 2:e6

doi:10.4081/es.2012.e6

duction mediating the cerebrovascular dysfunction induced by the amyloid -peptide and NADPH oxidase deficient mice exhibit reduced injury after stroke. ${ }^{19,20}$

In the present study, we used SH-SY5Y neuroblastoma cells to explore the role of NADPH oxidase $v s$. mitochondria-derived ROS in the development of diabetic neuropathy (DN) by using a specific NADPH oxidase inhibitor, gp91 ds-tat. The peptide-inhibitor gp91ds-tat prevents the assembly of NADPH oxidase subunits, thereby inhibiting the activity of the enzyme. Here we show that high glucose induced ROS is derived from NADPH oxidase and is involved in the apoptosis of SH-SY5Y neuroblastoma cells.

\section{Materials and Methods}

\section{Materials}

RPMI - 1640 glucose free medium, foetal bovine serum, penicillin, streptomycin, phosphate buffered saline (PBS), Cytochrome C, 
Diphenyleneiodonium (DPI), NADPH oxidase were purchased from Sigma (Poole, UK). Tissue culture plastics were obtained from Sarstedt (Leicester, UK) and Greiner Bio-one (Gloucestershire, UK). Carboxy- $\mathrm{H}_{2}$ DCFDA and MitoSOX ${ }^{\mathrm{TM}}$ were purchased from Invitrogen (Paisley, UK). NADPH oxidase inhibitor, gp91dstat scrambled and unscrambled was purchased from Anaspec (California, USA). Homogeneous Caspases assay was purchased from Roche Diagnostics (Indianapolis, IN, USA).

\section{Cell culture and treatment}

The human neuroblastoma cell line, SHSY5Y were maintained in RPMI-1640 medium supplemented with $5.5 \mathrm{mM}$ glucose, $20 \%$ fetal bovine serum, $100 \mathrm{U} / \mathrm{mL}$ penicillin, and 100 $\mathrm{U} / \mathrm{mL}$ streptomycin in a humid atmosphere of $5 \% \mathrm{CO}_{2}$ and $95 \% \mathrm{O}_{2}$ at $37^{\circ} \mathrm{C}$. SH-SY5Y cells were harvested using $0.25 \%$ trypsin-EDTA and cultured for 24 hours before being treated.

Cells were treated with normal glucose (NG, $5.5 \mathrm{mM}$ ), high glucose (HG, $25 \mathrm{mM}$ ) and mannitol (25 mM) to act as an osmotic control, for 6 days. Medium was changed every 2 days to maintain the amount of glucose in culture. To treat cells with the NADPH oxidase inhibitor (gp91ds-tat, scrambled and unscrambled, 1 $\mu \mathrm{M})$, the solution was prepared in HG medium. At the end of the incubation period, conditioned medium was removed and cells were washed with phosphate buffered saline (PBS) and stored at $-20^{\circ} \mathrm{C}$ until use.

\section{Assessments of caspase activity}

SH-SY5Y cells were cultured on black 96-well cell culture plates for $24 \mathrm{~h}$ before treatment. Cells were treated with NG (5.5 mM), HG (25 $\mathrm{mM}$ ) and mannitol ( $25 \mathrm{mM}$ ) for 6 days. Medium was changed every 2 days and at the end of the incubation period the caspase activity was assessed. To assess the activation of caspase proteases during apoptosis, a homogneous caspases activity kit was used which detect caspase 2, 3, 7 and caspase 6, 8, 9 and 10 to a lesser extent, the protocol was followed according to the manufacturer's instructions (Roche Applied Science, UK). Briefly, $100 \mu \mathrm{L}$ of caspase substrate (DEVD-Rhodamine 110) was added to each well containing $100 \mu \mathrm{L}$ of medium and the plate was incubated for $2 \mathrm{~h}$ at $37^{\circ} \mathrm{C}$. Upon the cleavage of the substrate by activated caspases, fluorescence of the released Rhodamine 110 was measured at excitation $485 \mathrm{~nm}$ and emission at $520 \mathrm{~nm}$ on PHERAstar fluorescence plate reader (BMG Labtech, Germany).

\section{Measurement of intracellular reactive oxygen species}

The fluorogenic probe, 6 - carboxy-2',7' dichlorofluorescein diacetate (Carboxy$\mathrm{H}_{2}$ DCFDA) was used to assess the formation of intracellular ROS. Carboxy- $\mathrm{H}_{2}$ DCFDA readily

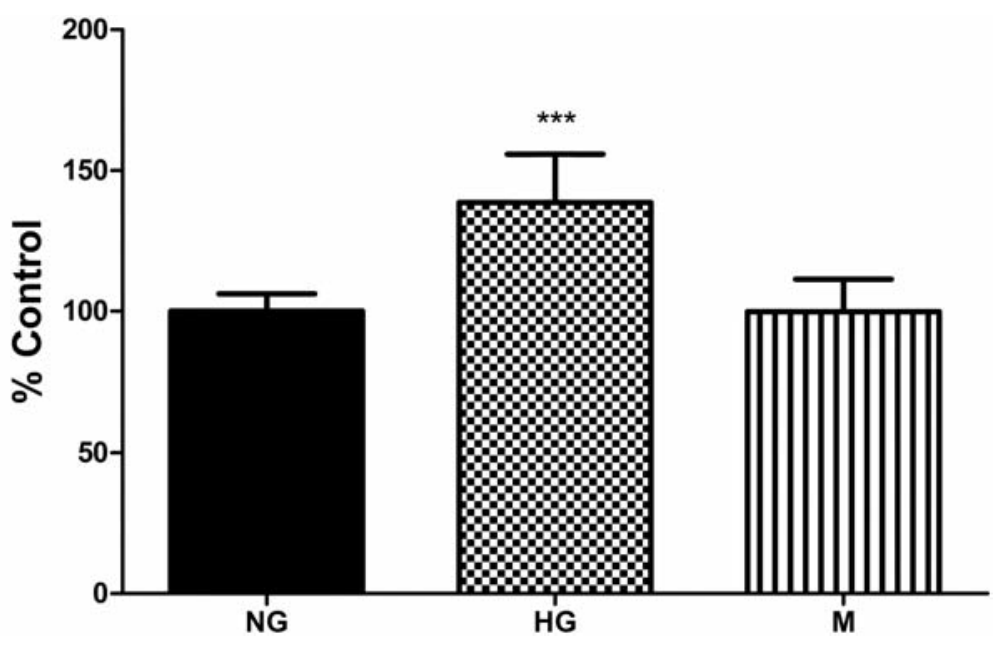

Figure 1. High glucose treatment increases caspases activity in SH-SY5Y cells. SH-SY5Y cells were treated with $100 \mathrm{~mL} /$ well of normal glucose (NG, $5.5 \mathrm{mM})$, high glucose (HG, $25 \mathrm{mM})$ and mannitol $(25 \mathrm{mM})$ for 6 days. At the end of the treatment, $100 \mu \mathrm{L}$ of the caspases substrate was added per well and the plate was incubated for $2 \mathrm{~h}$ at $37^{\circ} \mathrm{C}$. The fluorescence of Rhodamine 110 released from the cleavage of DEVD-Rhodamine 110 by activated caspases was measured. HG treatment increased caspase activity as compared to NG. Data was expressed as mean \pm standard deviation and presented as percentage of control of 3 separate experiments. ${ }^{* * *} \mathrm{P}<0.001$.

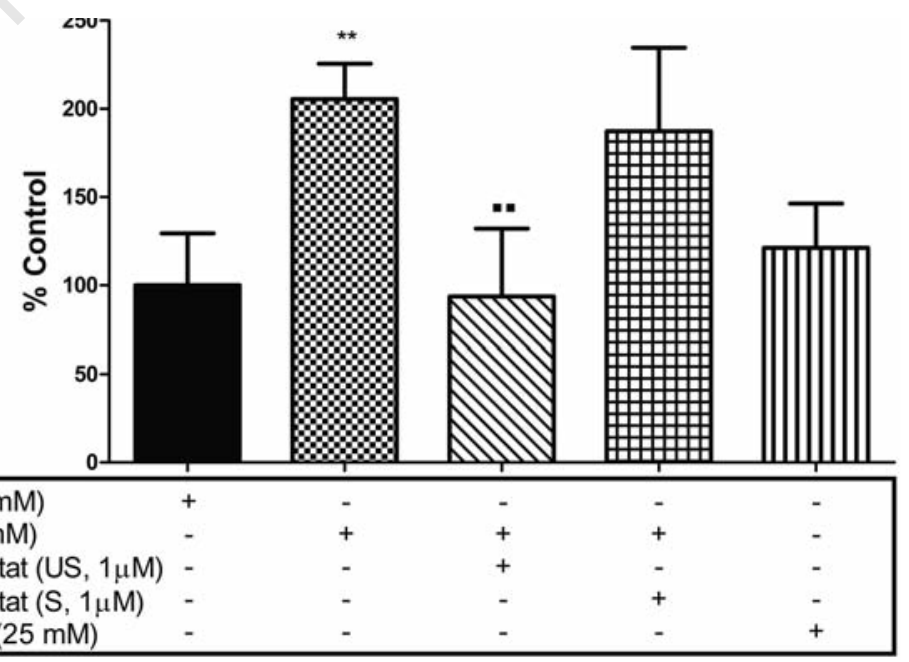

Figure 2. Nicotinamide adenine dinucleotide phosphate (NADPH) oxidase inhibitor reverses glucose induced reactive oxygen species (ROS) levels. SH-SY5Y cells were treated in 96-well plates with normal glucose (NG, $5.5 \mathrm{mM})$, high glucose (HG, $25 \mathrm{mM})$, mannitol $(25 \mathrm{mM})$ and the NADPH oxidase specific inhibitor gp91ds-tat $(1 \mu \mathrm{M}$, scrambled and unscrambled) prepared in HG medium for 6 days. Carboxy- $\mathrm{H}_{2}$ DCFDA (10 $\mu \mathrm{M})$ was added 30 minutes before the end of the treatment, medium was removed, cells washed and $100 \mu \mathrm{L} / w e l l$ of PBS was added and the fluorescence was measured at excitation $495 \mathrm{~nm}$ and the emission $525 \mathrm{~nm}$. HG treatment significantly increased intracellular ROS levels in SH-SY5Y cells compared to cells in NG $\left({ }^{* *} \mathbf{P}<0.01, n=3\right)$ and this increase in ROS levels was significantly reversed by the co-treatment of cells with the NADPH oxidase inhibitor gp91ds-tat $(n P<0.01, n=3)$. Data was expressed as mean \pm standard deviation and presented as percentage of control. 
enters cells and measures hydroxyl, peroxyl and other ROS. The probe is first deacetylated by esterases in the cytosol and this product is oxidized by ROS to a highly fluorescent entity, carboxy-2', 7'-dichlorofluorescin.

SH-SY5Y cells were cultured in clear bottom black 96 -well plates before being treated. Cells were treated with NG (5.5 mM), HG (25 mM) and mannitol to assess the formation of ROS formed under hyperglycaemic conditions. 30 min before the end of the treatment, $10 \mu \mathrm{M}$ carboxy- $\mathrm{H}_{2}$ DCFDA dissolved in cell culture medium was added to the cells. At the end of the treatment period, the media was removed and the cells were washed in PBS and $100 \mu \mathrm{L}$ of PBS was added in each well. The fluorescence was measured using a PHERAstar plate reader (BMG Labtech, Germany) with the excitation $485 \mathrm{~nm}$ and the emission $520 \mathrm{~nm}$.

\section{Measurement of mitochondrial superoxide levels and} mitochondrial membrane potential

Mitochondrial superoxide levels were analysed using MitoSOX ${ }^{\mathrm{TM}}$ red superoxide indicator. MitoSOX ${ }^{\mathrm{TM}}$ is live cell permeant and is rapidly and selectively targeted into the mitochondria and once in the mitochondria it is oxidized by superoxide and exhibits red fluorescence.

Tetramethyl Rhodamine Methyl Ester (TMRM) was used as a mitochondrial specific probe to measure the mitochondrial membrane potential $(\Delta \Psi \mathrm{m})$. TMRM is loaded into the cells via the plasma membrane and the dye subsequently sequesters into the mitochondria as the mitochondrial matrix is the most negatively charged part of the cell. Metabolically stressed cells with a loss of $\Delta \Psi \mathrm{m}$ exhibit a reduced level of fluorescence intensity.

30 minutes before the end of the treatment, MitoSOXTM $(5 \mu \mathrm{M})$ or TMRM $(1 \mu \mathrm{M})$ dissolved in cell culture medium were added to the cells. At the end of the treatment period, the media was removed and the cells were washed in PBS and $100 \mu \mathrm{L}$ of PBS was added in each well. To assess the mitochondrial superoxide levels using MitoSOX ${ }^{\mathrm{TM}}$, the fluorescence was measured at excitation $510 \mathrm{~nm}$ and the emission $580 \mathrm{~nm}$ and for mitochondrial membrane potential using TMRM, the fluorescence was measured at excitation $530 \mathrm{~nm}$ and emission $590 \mathrm{~nm}$ using a PHERAstar plate reader (BMG Labtech, Germany).

\section{Quantification of nicotinamide adenine dinucleotide phosphate oxidase activity}

Measurement of superoxide $\left(\mathrm{O}_{2}^{-}\right)$was based on the capacity of NADPH oxidase to reduce ferricytochrome $c$ in ferrocytochrome at $\mathrm{pH} 7.8$ as described earlier. ${ }^{21}$ Total cell lysate (25 mg protein/experiment), cytochrome c

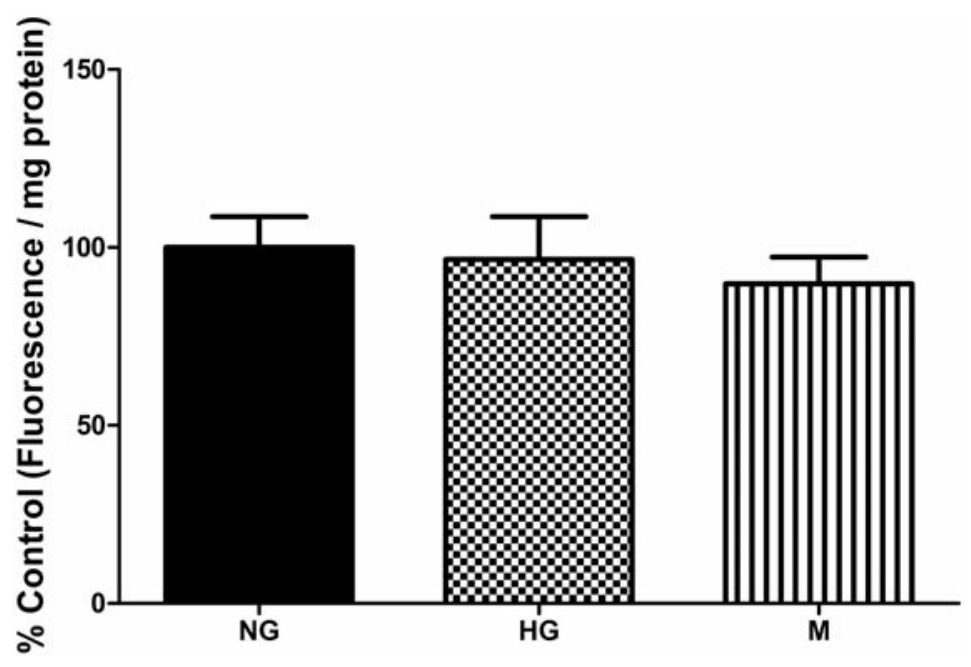

Figure 3. High glucose does not affect mitochondrial superoxide levels. SH-SY5Y cells were treated with normal glucose (NG, $5.5 \mathrm{mM}$ ), high glucose (HG, $25 \mathrm{mM}$ ) and mannitol for 6 days. MitoSOX ${ }^{\mathrm{TM}}(5 \mu \mathrm{M})$ was added $30 \mathrm{~min}$ before the end of the treatment and after the incubation, cells were washed with PBS and $100 \mathrm{~mL}$ of PBS added per well before the fluorescence was measured at excitation $510 \mathrm{~nm}$ and the emission $580 \mathrm{~nm}$. There was no significant difference between NG and HG $(n=3)$. Data expressed as mean \pm standard deviation and presented as percent of control (Fluorescence/mg protein).

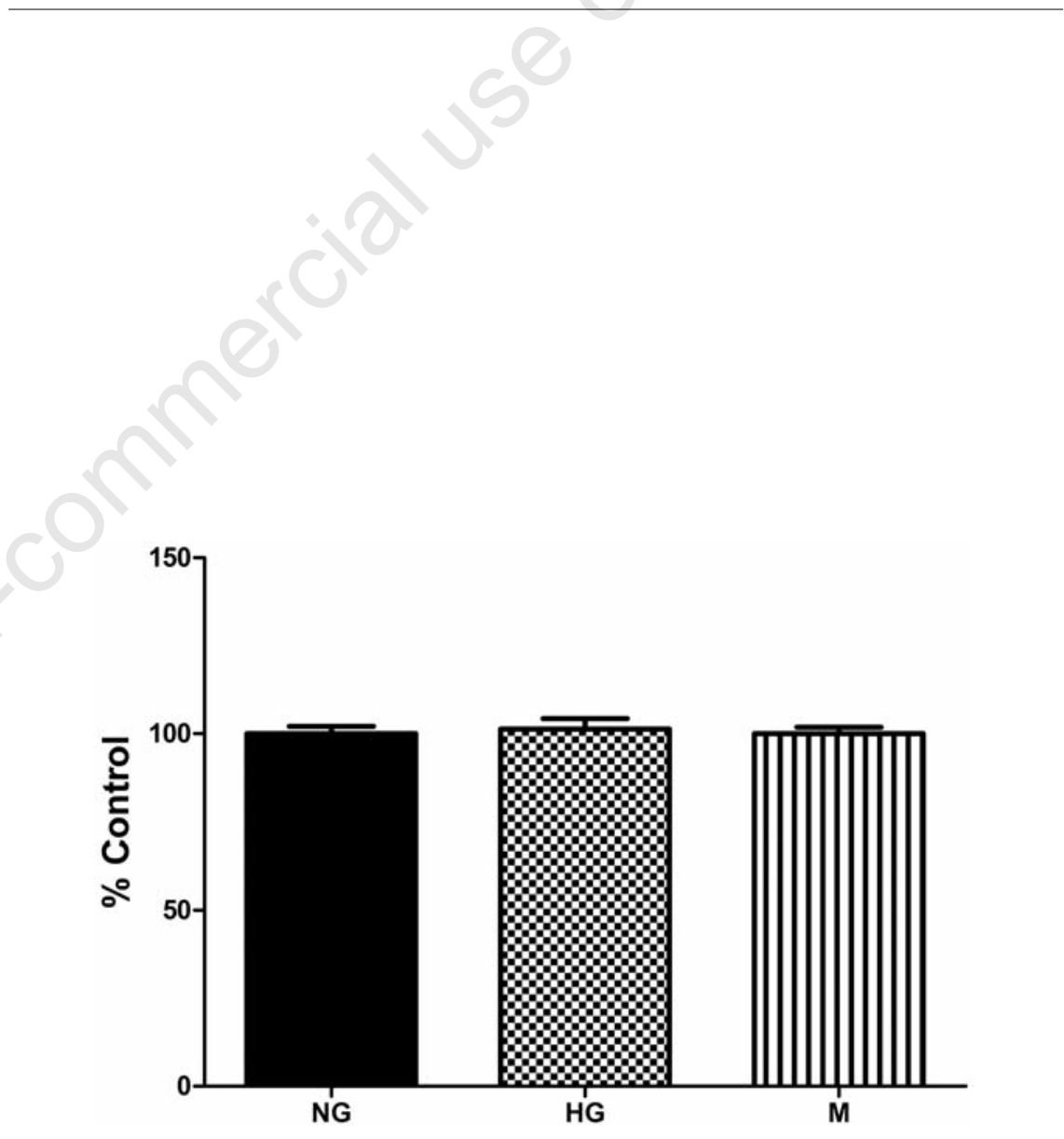

Figure 4. High glucose treatment does not affect the mitochondrial membrane potential of SH-SY5Y cells. SH-SY5Y cells were treated with normal glucose $(5.5 \mathrm{mM})$, high glucose $(25 \mathrm{mM})$ and mannitol $(25 \mathrm{mM})$ for 6 days. TMRM $(1 \mu \mathrm{M})$ was added $30 \mathrm{~min}$ before the end of the treatment and at the end of the treatment, cells were washed with PBS and $100 \mu \mathrm{L}$ of PBS added per well and the fluorescence was measured at excitation $520 \mathrm{~nm}$ and emission $560 \mathrm{~nm}$. There was no significant difference in the mitochondrial membrane potential in cells treated with HG compared to NG $(n=3)$. Data expressed as percent of control. 
(250 $\mathrm{mg} / \mathrm{L}$ final concentration) and NADPH $(100 \mathrm{mM})$ were incubated at $37^{\circ} \mathrm{C}$ for $120 \mathrm{~min}$, either in the presence or absence of diphenyleneiodonium (DPI, $100 \mathrm{mM}$ ), an NADPH oxidase inhibitor. The reduction of cytochrome $\mathrm{c}$ was calculated by measuring the absorbance at $550 \mathrm{~nm}$. $\mathrm{O}_{2-}^{-}$production in $\mathrm{nmol} / \mathrm{mg}$ protein was calculated from the difference between absorbance of reactions with and without DPI and the extinction coefficient $21 \mathrm{mmol} / \mathrm{L} / \mathrm{cm}$.

\section{Assessment of neurite extension and neurites per cell}

SH-SY5Y cells were cultured in 6-well cell culture plates for $24 \mathrm{hrs}$. Cells were treated with retinoic acid (RA, $10 \mu \mathrm{M})$ to induce neuronal differentiation in conjunction with $\mathrm{NG}$ (5.5 mM), HG (25 mM) and mannitol (25 mM). Cells were co-treated with gp91ds-tat (scrambled and unscrambled, $1 \mu \mathrm{M})$ in HG ( $25 \mathrm{mM}$ ) medium along with RA. At the end of the incubation period 10 random images were taken (Quantalab software, Nikon TS1000) and neurite-bearing cells were calculated from counts of 10 random fields. Image J was used to measure the length of the neurites.

\section{Protein measurement}

Total protein was measured using the BCA protein assay kit (Pierce, UK).

\section{Statistical analysis}

The statistical software Graph Pad Prism version 5.0 was used. One-way Analysis of Variance (ANOVA) and was used to test the significance between variables and a twotailed Student's $t$-test was used to test the significance of paired data. Data are expressed as means \pm standard deviation of measurements in the different experiments. Differences between treatment groups were considered statistically significant at $\mathrm{P}<0.05$.

\section{Results}

\section{High glucose induces apoptosis in}

\section{SH-SY5Y cells}

The effect of high glucose (HG) on SH-SY5Y cell viability was determined using the Homogeneous Caspase assay kit (Roche, UK). As shown in Figure 1, chronic treatment of SHSY5Y cells with HG ( $25 \mathrm{mM})$ for 6 days resulted in a significant increase in the caspase activity as compared to $\mathrm{NG}(5.5 \mathrm{mM})$ $(138.60 \pm 17.25 \%$ versus $100.0 \pm 6.18 \% \mathrm{P}<0.001$, $\mathrm{n}=3$ ). Mannitol, an osmotic control had no effect on apoptosis as compared to NG $(99.98 \pm 11.52 \%$ versus $100.00 \pm 6.18 \%, \mathrm{n}=3)$.
Inhibiting nicotinamide adenine dinucleotide phosphate oxidase reversed the glucose-induced increase in reactive oxygen species

Intracellular ROS levels were significantly increased in SH-SY5Y cells treated with HG (25 mM) for 6 days as compared to NG (5.5 $\mathrm{mM})$ treated cells $(205.5 \pm 29.4 \%$ versus $100.0 \pm 17.7 \%(\mathrm{P}<0.01, \mathrm{n}=3)$ (Figure 2$)$.

As seen in Figure 2, co-treatment of cells with the inhibitor of NADPH oxidase, GP91 dstat (unscrambled, $1 \mu \mathrm{M}$ ) reversed the increase in HG induced ROS formation $(93.63 \pm 38.37 \%$ versus $205.5 \pm 20.05 \%, \mathrm{P}<0.01, \mathrm{n}=3$ ).

\section{Mitochondrial reactive oxygen} species levels remained unchanged after exposure to high glucose

Exposure to HG (25 mM) for 6 days did not change the levels of mitochondrial superoxide production in SH-SY5Y cells (Figure 3) $(96.63 \pm 12.05 \%$ compared to normal glucose $100.0 \pm 8.7 \%, n=3)$.
High glucose treatment did not alter mitochondrial membrane potential

HG treatment did not alter the mitochondrial membrane potential of SH-SY5Y cells (Figure 4) as compared to NG (5.5 mM) after exposure for 6 days $(101.3 \pm 2.9 \%$ compared to $100.0 \pm 2.0 \%, n=3)$.

\section{Glucose induced an increase in}

nicotinamide adenine dinucleotide phosphate oxidase activity

SH-SY5Y cells under HG (25 mM) conditions for 6 days increased the superoxide production compared to cells treated with NG $(5.5 \mathrm{mM})$ $(0.13 \pm 0.04 \mathrm{mM} / \mathrm{mg}$ protein versus $0.03 \pm 0.03$ $\mathrm{mM} / \mathrm{mg}$ protein). The co-treatment of cells with the NADPH oxidase specific inhibitor, gp91ds-tat reversed the increase in the superoxide production $(0.04 \pm 0.04 \mathrm{mM} / \mathrm{mg}$ protein versus $0.13 \pm 0.04$ $\mathrm{mM} / \mathrm{mg}$ protein). The scrambled gp91ds-tat did not alter the increase in superoxide production $(0.12 \pm 0.06 \mathrm{mM} / \mathrm{mg}$ protein versus $0.13 \pm 0.04$ $\mathrm{mM} / \mathrm{mg}$ protein) in SH-SY5Y cells (Figure 5).
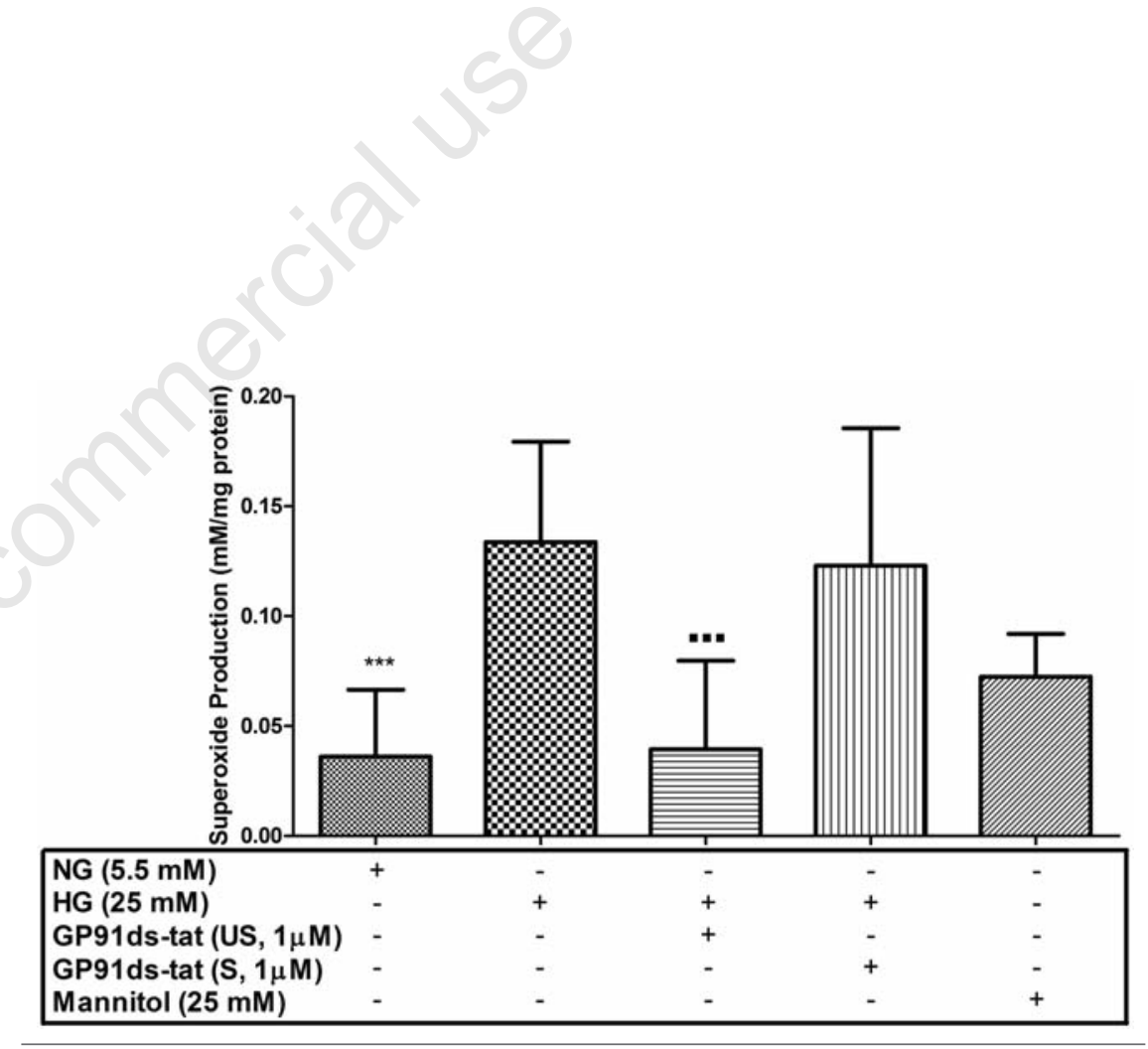

Figure 5. High glucose increases nicotinamide adenine dinucleotide phosphate (NADPH) oxidase activity. SH-SY5Y cells were cultured in $30 \mathrm{~mm}^{2}$ dishes were exposed to normal glucose (NG, $5.5 \mathrm{mM})$ and high glucose $(\mathrm{HG}, 25 \mathrm{mM})$ in the absence and presence of the specific NADPH oxidase inhibitor, gp91 ds-tat $(1 \mu \mathrm{M})$ for 6 days and lysed. Lysates $(50 \mu \mathrm{g}$ protein), cytochrome c $(250 \mu \mathrm{g} / \mathrm{L}$ final concentration) and NADPH $(100 \mu \mathrm{M})$ were incubated at $37^{\circ} \mathrm{C}$ for $120 \mathrm{~min}$, either in the presence or absence of diphenyleneiodonium (DPI, $100 \mu \mathrm{M})$. The reduction of cytochrome c was measured by reading the absorbance at 550 $\mathrm{nm}$. Superoxide $\left(\mathrm{O}_{2}^{-}\right)$production in $\mathrm{mmol} / \mu \mathrm{g}$ protein was calculated from the difference between absorbance of samples at 0 and $120 \mathrm{~min}$ and the extinction coefficient $21 \mathrm{mmol} / 1 / \mathrm{cm}$. Exposure to HG significantly increases NADPH oxidase $(* * * P<0.001$ as compared to NG, $n=6-14$ ) activity which is reversed by the addition of the specific NADPH oxidase inhibitor gp91 ds-tat $(1 \mu \mathrm{M})\left({ }^{* * *} \mathbf{P}<0.001\right.$ as compared to $\left.\mathrm{HG}, \mathbf{n}=6-14\right)$. Data is presented as mean \pm standard deviation of 6-14 separate experiments. 


\section{High glucose reduces neurite} extension and neuritis per cell

SH-SY5Y cells treated with HG (25 mM) for 6 days had a marked reduction in the neurite outgrowth (Figure 6A) $(151.00 \pm 44.02$ pixels versus to $285.00 \pm 71.98$ pixels). In addition, the number of neurites per cell was also significantly lower than cells treated with $\mathrm{NG}$ ( $2.28 \pm 0.63$ neurites compared to $3.65 \pm 0.68$ neurites) (Figure 6B). Co-treating the cells with the NADPH oxidase inhibitor gp91 ds-tat (1 $\mu \mathrm{M}$ unscrambled) prevented the inhibition in neurite outgrowth under $\mathrm{HG}$ treatment $(331 \pm 99.46$ pixels compared to $151.0 \pm 44.02$ pixels) and neurites per cell $(3.48 \pm 0.59$ neurites compared to $2.28 \pm 0.63$ neurites).

\section{Discussion}

It is now widely accepted that increased oxidative stress, the imbalance between the production of ROS and the antioxidant capability of the cell, plays a key role in the pathogenesis of DN.22,23 The possible sources for ROS in diabetes include enzymatic pathways, auto-oxidation of glucose, and the mitochondria. 7,24 The mitochondrial-dependent processes are considered as a central mediator of oxidative stress in diabetic complications and normalizing mitochondrial ROS prevents glucose induced activation of biochemical pathways involved in the pathogenesis of DN. ${ }^{15}$ Antioxidants have been shown to prevent the symptoms of DN in experimental systems. ${ }^{25,26}$ We have recently suggested the role for NADPH oxidase and not mitochondria derived ROS (mtROS) in DR. In the present study, we explore the role of NADPH oxidase vs. mitochondrial-derived ROS in the development of DN.

Impairment in signalling mechanisms regulating neuron differentiation is hypothesised to be a main cause of neuronal dysfunction and neurite outgrowth was shown to be significantly retarded in diabetic neurons of streptozotocin induced diabetic rats. ${ }^{10,27}$ Elevated glucose increases oxidative stress and inhibits neurite outgrowth in retinoic acid promotion of neurite outgrowth in cortical neurons. ${ }^{10}$ Moreover, recent studies have demonstrated that high glucose induces apoptosis in primary dorsal root ganglion neurons, ${ }^{7}$ PC12 cells and SH-SY5Y human neuroblastoma cells. ${ }^{28,29}$ Consistent with these findings, the present study demonstrates that exposure of SH-SY5Y cells to HG for 6 days causes increased caspase activity. Increased apoptosis in diabetic neurons involves decreased levels of $\mathrm{Bcl}-2$ and translocation of mitochondrial cytochrome $\mathrm{C}$ to the cytosol. ${ }^{30}$ The induction of apoptosis under HG conditions has also been attributed to the increased production of ROS.16,31
A)

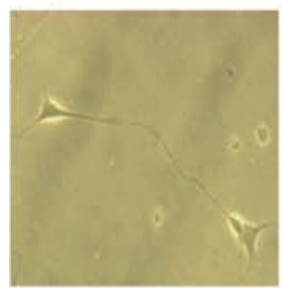

NG+RA

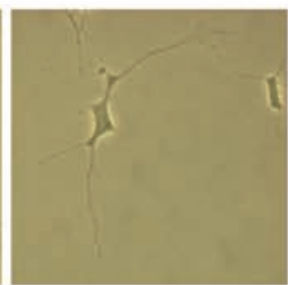

HG+RA

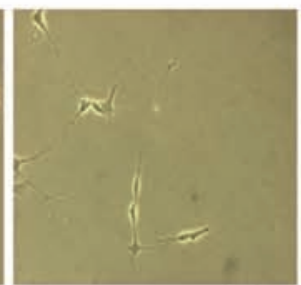

HG+gp91ds-tat (US)

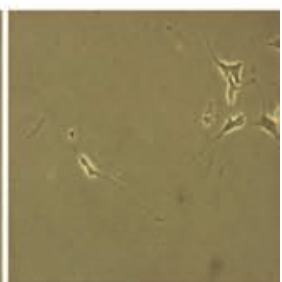

HG+gp91ds-tat (S)

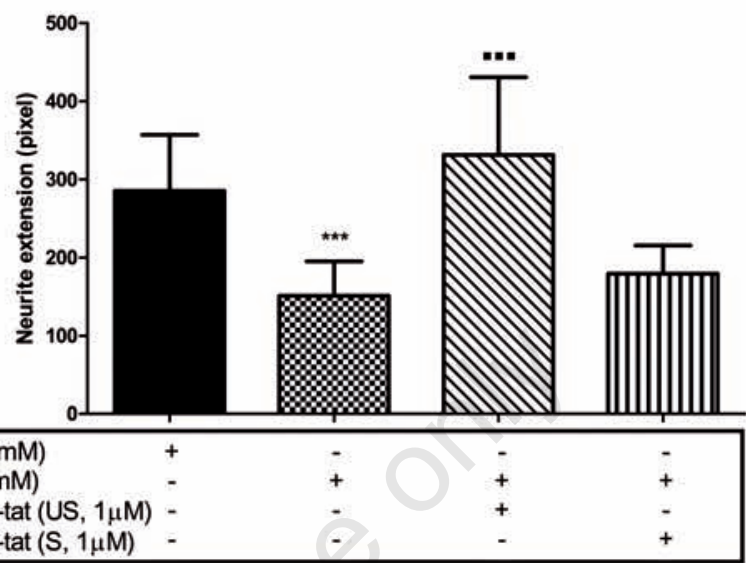

B)

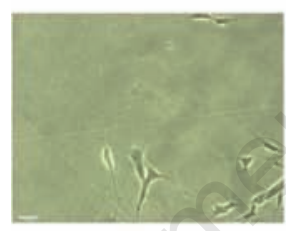

NG+RA

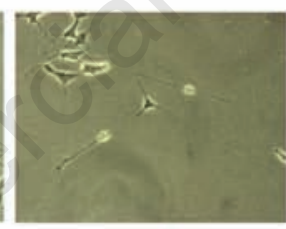

HG+RA

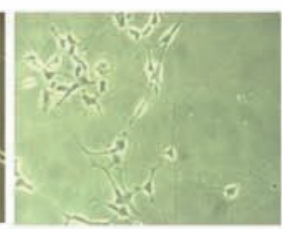

HG+gp91ds-tat (US)

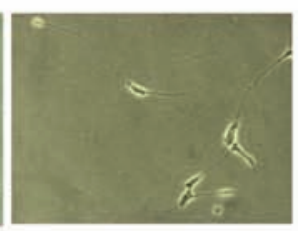

HG+gp91ds-tat (S)

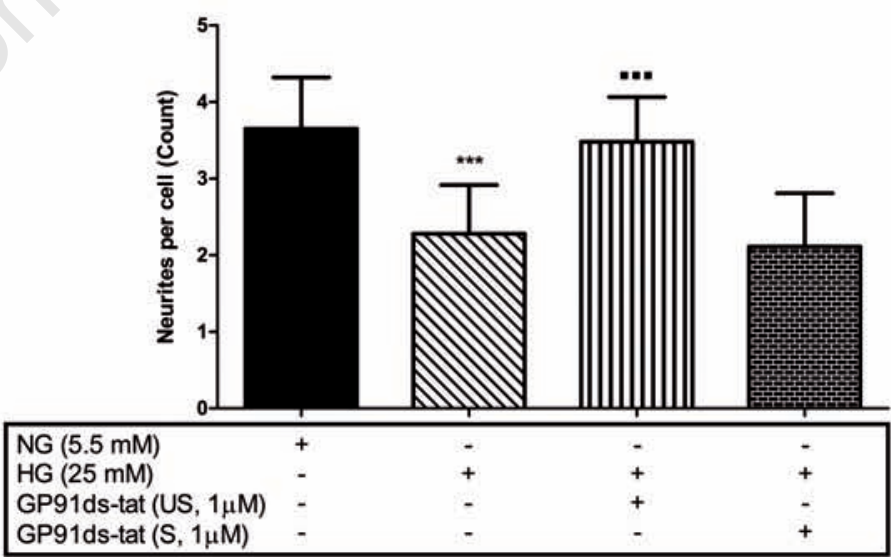

Figure 6. High glucose treatment inhibits neurite extension and number of the neurites per cell. SH-SY5Y cells were cultured in 6 -well plates and treated with normal glucose (NG, $5.5 \mathrm{mM})$ or high glucose $(\mathrm{HG}, 25 \mathrm{mM})$ and co-treated with retinoic acid (RA, $\mathrm{X}$ $\mu \mathrm{M})$ to differentiate the cells. 10 random images were taken and neurite outgrowth was measured and counted using the imageJ software. HG treatment of cells showed a significant reduction in length of the neurite outgrowth (***P<0.001 as compared to NG) (A) and the number of neurites per cell $(* * * \mathrm{P}<\mathbf{0 . 0 0 1}$ as compared to NG) $(\mathrm{B})$. This reduction was reversed by the addition of the specific NADPH oxidase inhibitor gp91 ds-tat $(1 \mu \mathrm{M})$ in HG treatment ( $\mathrm{HI}<\mathbf{P . 0 0 1}$ as compared to HG). Data is presented as mean \pm standard deviation of 10 random images from 3 separate experiments and expressed as percent of control (Pixels). 
NADPH oxidase is a membrane bound enzyme complex consisting of two membranebound components (gp91 ${ }^{\text {PHOX }}$ and $\mathrm{p} 22^{\text {PHOX }}$ ), three components in the cytosol (p67 РHOX, p47 ${ }^{\text {PHOX }}$ and $\mathrm{p} 40^{\text {РнОХ }}$ ) and a low molecular weight $\mathrm{G}$ protein (either rac 2 or rac 1). ${ }^{32}$ Activation of NADPH oxidase is associated with, and caused by, the migration of the cytosolic components to the cell membrane so that the complete oxidase complex can be assembled. ${ }^{32}$ In the present study, the increased ROS induced by high glucose was significantly reversed by the NADPH oxidase specific inhibitor gp91ds-tat. This is consistent with our recent study involving retinal capillary pericytes as a model of early DR and highlights the important role of NADPH oxidase activity in ROS production under chronic hyperglycaemia. ${ }^{16}$ Dorsal root ganglion of diabetic rats have been shown to have an increased ROS production coupled with the loss of $\Delta \Psi \mathrm{m}^{7}$ ? n the contrary, our findings suggest that, mitochondrial superoxide production and mitochondrial membrane potential under HG conditions was unaffected in SH-SY5Y cells. Mitochondrial dysfunction (increased mitochondrial ROS and loss of $\Delta \Psi \mathrm{m})$ has been attributed to high glucose induced neuronal and Schwann cell death, 7,31 but here we report that neuronal cells exposed to chronic HG produced ROS from a non-mitochondrial source, NADPH oxidase. This difference could be attributed to the length of the exposure and the concentration of glucose-induced treatment. NADPH oxidase could be a key driver under chronic hyperglycaemic conditions.

Neurite outgrowth is a key event of neuronal differentiation and forms the basis of the proper connectivity within neuronal networks. ${ }^{33} \mathrm{SH}$ SY5Y cells possess a neuronal phenotype and are extensively used as a cellular model to study neuronal differentiation and neurite outgrowth. ${ }^{34,35}$ SH-SY5Y cells when treated with Alltrans-retinoic acid (RA), a physiologically active metabolite of vitamin A, exhibit this neuronal phenotype. ${ }^{36}$ RA promotes the activation of PI3K/AKT pathway, which is shown to be involved in neuronal survival and differentiation of SH-SY5Y cells and decreases hyperglycaemiainduced oxidative stress. ${ }^{10,37}$ Here we also report that exposure of SH-SY5Y cells to high glucose for 6 days caused a significant reduction in neurite outgrowth and neurites per cell. This is consistent with previous reports in cortical neurons, ${ }^{10}$ sympathetic superior mesenteric ganglion, superior cervical ganglion and dorsal root ganglion. ${ }^{38,39}$ The inhibition of neurite outgrowth in cortical neurons has been attributed to increased oxidative stress under hyperglycaemic conditions and in Neuro 2A neuroblastoma cells to the production of oxidative stress. ${ }^{10,40}$ This inhibition of neurite outgrowth seen in SH-SY5Y cells under hyperglycaemic conditions was reversed by the co-treatment of cells with gp91ds-tat.

\section{Conclusions}

In summary, our data i) highlights the importance of ROS from NADPH oxidase and not the mitochondria in glucose-induced increase in oxidative stress, loss of cell viability and inhibition of neurite outgrowth and neurites per cells, and ii) supports the beneficial effects of antioxidants to prevent oxidative stress and caspases-dependent apoptosis of SH-SY5Y cells. Our findings suggest that NADPH oxidase activity may be a therapeutic target for the treatment of progressive diabetic neuropathy.

\section{References}

1. Kumar A, Kaundal RK, Iyer S, Sharma SS. Effects of resveratrol on nerve functions, oxidative stress and DNA fragmentation in experimental diabetic neuropathy. Life Sci 2007;80:1236-44.

2. Boulton AJM, Malik RA, Arezzo JC, Sosenko JM. Diabetic somatic neuropathies. Diabetes Care 2004;27:1458-86.

3. Said G. Diabetic neuropathy - a review. Nat Clin Pract Neurol 2007;3:331-40.

4. UKPDS. Tight blood pressure control and risk of macrovascular and microvascular complications in type 2 diabetes: UKPDS 38. BMJ 1998;317:703-13.

5. DCCT. Early worsening of diabetic retinopathy in the diabetes control and complications trial. Arch 0phthalmol 1998; 116:874-86.

6. Brownlee M. The pathobiology of diabetic complications. Diabetes 2005;54:1615-25.

7. Russell JW, Golovoy D, Vincent AM, et al. High glucose-induced oxidative stress and mitochondrial dysfunction in neurons. FASEB J 2002;16:1738-48.

8. Schmeichel AM, Schmelzer JD, Low PA. Oxidative injury and apoptosis of dorsal root ganglion neurons in chronic experimental diabetic neuropathy. Diabetes 2003; 52:165-71.

9. Annunziato L, Amoroso S, Pannaccione A, et al. Apoptosis induced in neuronal cells by oxidative stress: role played by caspases and intracellular calcium ions. Toxicol Lett 2003;139:125-33.

10. Guleria RS, Pan J, DiPette D, Singh US. Hyperglycemia inhibits retinoic acidinduced activation of racl, prevents differentiation of cortical neurons, and causes oxidative stress in a rat model of diabetic pregnancy. Diabetes 2006;55:3326-34.

11. Li S-H, Cheng AL, Li H, Li X-J. Cellular defects and altered gene expression in PC12 cells stably expressing mutant huntingtin. J Neurosci 1999;19:5159-72.
12. Griendling KK, Sorescu D, Ushio-Fukai M. $\mathrm{NAD}(\mathrm{P}) \mathrm{H}$ oxidase: role in cardiovascular biology and disease. Circ Res 2000;86:494501.

13. Onozato ML, Tojo A, Goto A, et al. Oxidative stress and nitric oxide synthase in rat diabetic nephropathy: effects of ACEI and ARB. Kidney Int 2002;61:186-94.

14. Turrens JF. Mitochondrial formation of reactive oxygen species. J Physiol 2003; 552:335-44.

15. Nishikawa T, Edelstein D, Brownlee M. The missing link: a single unifying mechanism for diabetic complications. Kidney Int 2000;58:S26-S30.

16. Mustapha NM, Tarr JM, Kohner EM, Chibber R. NADPH oxidase versus mitochondria-derived ros in glucose-induced apoptosis of pericytes in early diabetic retinopathy. J Ophthalmol 2010;2010.

17. Al-Shabrawey M, Rojas M, Sanders T, et al. Role of NADPH oxidase in retinal vascular inflammation. Invest Ophthalmol Vis Sci 2008;49:3239-44.

18. Tojo A, Asaba K, Onozato ML. Suppressing renal NADPH oxidase to treat diabetic nephropathy. Expert Opin Ther Targets 2007;11:1011-8.

19. Park L, Anrather J, Zhou P, et al. NADPH oxidase-derived reactive oxygen species mediate the cerebrovascular dysfunction induced by the amyloid $\hat{\mathrm{I}}^{2}$ peptide. $\mathrm{J}$ Neurosci 2005;25:1769-77.

20. Walder CE, Green SP, Darbonne WC, et al. Ischemic stroke injury is reduced in mice lacking a functional NADPH oxidase. Stroke 1997;28:2252-8.

21. Miller Jr FJ, Griendling KK, Chandan K. Sen LP. Functional evaluation of nonphagocytic NAD(P)H oxidases. Methods Enzymol 2002;353:220-33.

22. Cotter MA, Cameron NE. Effect of the $\mathrm{NAD}(\mathrm{P}) \mathrm{H}$ oxidase inhibitor, apocynin, on peripheral nerve perfusion and function in diabetic rats. Life Sci 2003;73:1813-24.

23. Vincent AM, Russell JW, Low P, Feldman EL. Oxidative stress in the pathogenesis of diabetic neuropathy. Endocr Rev 2004;25: 612-28.

24. Wolff SP, Jiang ZY, Hunt JV. Protein glycation and oxidative stress in diabetes mellitus and ageing. Free Radic Biol Med. 1991;10:339-52.

25. Love A, Cotter MA, Cameron NE. Effects of the sulphydryl donor $\mathrm{N}$-acetyl-L-cysteine on nerve conduction, perfusion, maturation and regeneration following freeze damage in diabetic rats. Eur J Clin Invest 1996;26:698-706. Epub 1996/08/01.

26. Zherebitskaya E, Akude E, Smith DR, Fernyhough P. Development of selective axonopathy in adult sensory neurons isolated from diabetic rats. Diabetes 2009;58: 1356-64. 
27. Sakaue Y, Sanada M, Sasaki T, et al. Amelioration of retarded neurite outgrowth of dorsal root ganglion neurons by overexpression of PKCdelta in diabetic rats. Neuroreport 2003;14:431-6.

28. Sharifi AM, Mousavi SH, Farhadi M, Larijani B. Study of high glucose-induced apoptosis in PC12 cells: role of bax protein. J Pharmacol Sci 2007;104:258-62.

29. Serra-Pérez A, Verdaguer E, Planas AM, Santalucía T. Glucose promotes caspasedependent delayed cell death after a transient episode of oxygen and glucose deprivation in SH-SY5Y cells. J Neurochem 2008;106:1237-47.

30. Srinivasan S, Stevens M, Wiley JW. Diabetic peripheral neuropathy: evidence for apoptosis and associated mitochondrial dysfunction. Diabetes 2000;49:1932-8.

31. Vincent AM, Brownlee M, Russell JW. Oxidative stress and programmed cell death in diabetic neuropathy. Ann N Y Acad Sci 2002;959:368-83.
32. Babior BM. NADPH oxidase. Curr Opin Immunol 2004;16:42-7.

33. Da Silva JS, Dotti CG. Breaking the neuronal sphere: regulation of the actin cytoskeleton in neuritogenesis. Nat Rev Neurosci 2002;3:694-704.

34. Encinas M, Iglesias M, Liu $\mathrm{Y}$, et al. Sequential treatment of SH-SY5Y cells with retinoic acid and brain-derived neurotrophic factor gives rise to fully differentiated, neurotrophic factor-dependent, human neuron-like cells. J Neurochem 2000; 75:991-1003.

35. Miloso M, Villa D, Crimi M, et al. Retinoic acid-induced neuritogenesis of human neuroblastoma SH-SY5Y cells is ERK independent and PKC dependent. J Neurosci Res 2004;75:241-52.

36. Thiele CJ, Reynolds CP, Israel MA. Decreased expression of $\mathrm{N}$-myc precedes retinoic acid-induced morphological differentiation of human neuroblastoma. Nature 1985;313:404-6.
37. López-Carballo G, Moreno L, Masiá S, et al. Activation of the phosphatidylinositol 3kinase/akt signaling pathway by retinoic acid is required for neural differentiation of sh-sy5y human neuroblastoma cells. J Biol Chem 2002;277:25297-304.

38. Semra YK, Smith NCE, Lincoln J. Comparative effects of high glucose on different adult sympathetic neurons in culture. Neuroreport 2004;15:2321-5.

39. Tosaki T, Kamiya H, Yasuda Y, et al. Reduced NGF secretion by Schwann cells under the high glucose condition decreases neurite outgrowth of DRG neurons. Exp Neurol 2008;213:381-7.

40. Neely MD, Sidell KR, Graham DG, Montine TJ. The lipid peroxidation product 4hydroxynonenal inhibits neurite outgrowth, disrupts neuronal microtubules, and modifies cellular tubulin. J Neurochem 1999;72:2323-33. 